\title{
Extraction of Psychological Symptoms and Instantaneous Respiratory Frequency as Indicators of Internet Addiction Using Rule-Based Machine Learning
}

\author{
Hung-Ming Chi ${ }^{1}$, Liang-Yu Chen ${ }^{2}$, Tzu-Chien Hsiao ${ }^{1,3, *}$ \\ ${ }^{1}$ Department of Computer Science, College of Computer Science, National Yang Ming Chiao Tung University, Hsinchu, 300, Taiwan \\ ${ }^{2}$ Institute of Computer Science and Engineering, College of Computer Science, National Yang Ming Chiao Tung University, Hsinchu, \\ 300, Taiwan
}

${ }^{3}$ Institute of Biomedical Engineering, College of Electrical and Computer Engineering, National Yang Ming Chiao Tung University, Hsinchu, 300, Taiwan

\begin{tabular}{l} 
A R T I C L E I N F O \\
\hline Article history: \\
Received: 20 July, 2021 \\
Accepted: 25 September, 2021 \\
Online: 30 September, 2021
\end{tabular}

Keywords:

Extended Classifier System

Behavioral Addiction

Psychological Symptom

Instantaneous Frequency

\begin{abstract}
A B S T R A C T
Internet addiction (IA) has adverse effects on psychophysiological responses, interpersonal relationships, and academic and occupational performance. IA detection has received increasing attention. Although questionnaires enable long-term assessment (over 6 months) and physiological measurements to aid the short-term evaluation (over 2 min) of IA, the lack of algorithms results in an inability to detect IA in real time. A computer-aided system can address this problem. This study used the extended classifier system with continuous realcoded variables (XCSR) for rule-based machine learning to classify IA risk. Chen Internet Addiction Scale (CIAS) items were verified and instantaneous respiratory features of IA were extracted with "don't care" attribute values. The result demonstrated that the XCSR model achieved more than 95\% classification accuracy. Using the "don't care" attribute values, the CIAS items were reduced from 26 to 19, and the instantaneous frequency (IF) of respiratory muscle contractions, respiratory wall movements, and body movements were extracted as IA-related features. These findings suggested that the XCSR model is a potentially useful system for detecting IA. The modified 19-item CIAS and IF of respiration can be adopted to assist in the real-time detection of IA and explore the psychophysiological developments of IA users. In future studies, more samples must be collected to validate these findings and instantaneous physiological responses investigated with different window sizes while participants with IA engage in active online gameplay.
\end{abstract}

\section{Introduction}

This paper constitutes a continuation of the work originally presented at the 2019 Genetic and Evolutionary Computation Conference [1]. The Internet has changed our daily lives profoundly over the last three decades. People now have easy access to the Internet by using desktops, smartphones, tablets, laptops, and other digital devices, through which they interact with friends, purchase products, play games, search for information, and send emails. Despite the convenience the Internet lends to our lives, some serious concerns remain.

Some individuals intensely indulge in and depend on the Internet, which can lead to negative ramifications, such as

${ }^{*}$ Corresponding Author: Tzu-Chien Hsiao, 705R, MIRC, 1001 University Road, Hsinchu, Taiwan, labview@g2.nctu.edu.tw

www.astesj.com

https://dx.doi.org/10.25046/aj060522 estrangement from family and friends, impaired mental and physical health, and low academic and work performance [2, 3]. This condition has been termed Internet addiction (IA) [4], and it has various subtypes, including problematic online game addiction [5], problematic cellphone use [6], and social network mental disorder [7]. Scholars have reported that problematic game use in particular was associated with severe clinical impairment, and Internet gaming disorder (IGD) has been preliminarily listed in the Diagnostic and Statistical Manual of Mental Disorders (DSM), Fifth Edition [8]. A 2019 announcement by the World Health Organization recognized gaming disorder as a mental disorder [9]. The IA prevalence rate in Asia [3], Europe [10], and the United States [11] was $5.6 \%, 7.97 \%$, and $8.1 \%$, respectively, with the risk of developing IA increasing as a result of the 2019 coronavirus 
disease pandemic $[12,13]$. IA is a common behavioral addiction globally and continues to receive considerable attention.

Psychological symptoms and physiological responses are key indicators for detecting addiction behavior. IA-related questionnaires are used to evaluate the symptoms of IA, but they have some limitations. First, different questionnaires must be designed that correspond to various cultures and environments worldwide. The question of how many psychological symptoms must be incorporated into an IA questionnaire remains debated. Second, questionnaires are typically adopted to assess Internet experiences over a long-term period (over 6 months) [2, 3, 5, 8, 9, 14-18] and are not ideal for examining short-term or current experiences. Third, Internet users must spend time completing these questionnaires. Fourth, subjective experiences can affect the assessment of IA. Researchers can potentially observe the physiological responses of Internet users alongside questionnaires to assist in the assessment of IA risk. In such assessments, the major physiological signals are analyzed or calculated using descriptive statistics or generic mathematics [10, 19-23], although these algorithms may be insufficient to estimate the dynamical and instantaneous nature of physiological responses. Some studies have collected user behavior or social media and online gaming records to input into machine learning systems to identify addiction behaviors [7, 24-28]. However, this type of data collection is time consuming, and the real-time detection of IA presents a considerable challenge.

The extended classifier system (XCS) method is a rule-based machine learning system that assumes an agent role to interact with the environment and produce the optimum policy [29]. The XCS method generates rules with each interaction, each in the form of an "IF condition THEN action." The conditions in the rules employ coded symbols $\{0,1, \#\}$; the symbol \# involves ignoring some values and is also referred to as the "don't care" attribute. The XCS method is applied to manage binary problems, and when combined with the continuous real-coded variables (XCSR) method, it can solve real-value problems [30]. Unlike other machine learning systems, the learning process of the XCSR method can be observed directly, and the "don't care" attribute can extract the main features for classification. Therefore, this study adopted the XCSR method as a computer-aided system to detect the risk of IA using Chen Internet Addiction Scale (CIAS) items [3] as input data. The XCSR method was then applied to verify the major psychological symptoms of IA from the CIAS data. To investigate the instantaneous physiological responses of IA, the ensemble empirical mode decomposition (EEMD) method [31] was used to decompose a physiological signal into instantaneous components, and the XCSR method was employed to extract the IA-related physiological features from these components. This study has two main contributions, namely the use of a rule-based machine learning system to classify the risk of IA, and the extraction of the major symptoms and instantaneous physiological features of IA from a questionnaire and from physiological components, respectively. These developments may be used to design a new short-form IA questionnaire and instantaneous physiological model to detect IA in real time. These extracted psychological symptoms and instantaneous responses can assist in exploring the developmental process of IA behavior.
The organization of this paper is as follows: The Literature Review section outlines the psychological symptoms covered in the IA questionnaire, physiological responses of the IA group, and detection of behavioral addiction using machine learning systems. The Research Methodology section presents the overview of the XCSR and EEMD methods, and the Experiment section describes the design of experiment and data analysis. The Results section reveals the classification accuracy of the XCSR using the CIAS items and instantaneous physiological waveforms as input data, with the rate of non-“don't care” values calculated for these input data. In the Discussion section, the study findings and limitations are discussed, and the Conclusion section summarizes the study.

\section{Literature Review}

\subsection{IA questionnaires}

The design of IA questionnaires is based on substance abuse, substance dependence, and pathological gambling criteria from the DSM, Fourth Edition, IGD criteria from the DSM, Fifth Edition, and gaming disorder criteria from the International Classification of Diseases, Eleventh Revision. IA questionnaires cover various psychological symptoms (Table 1), including abandoning other activities $\left(\mathrm{S}_{\mathrm{A}}\right)$, craving $\left(\mathrm{S}_{\mathrm{C}}\right)$, compulsive symptoms $\left(\mathrm{S}_{\mathrm{CS}}\right)$, deceit or cover-up $\left(\mathrm{S}_{\mathrm{DC}}\right)$, health-related problems $\left(\mathrm{S}_{\mathrm{H}}\right)$, interpersonal problems $\left(\mathrm{S}_{\mathrm{I}}\right)$, mood modification $\left(\mathrm{S}_{\mathrm{MM}}\right)$, preoccupation or salience $\left(\mathrm{S}_{\mathrm{pS}}\right)$, school or occupational problems $\left(\mathrm{S}_{\mathrm{so}}\right)$, tolerance $\left(\mathrm{S}_{\mathrm{T}}\right)$, time management problems $\left(\mathrm{S}_{\mathrm{TM}}\right)$, and withdrawal symptoms $\left(\mathrm{S}_{\mathrm{ws}}\right)$. Researchers have debated how many psychological symptoms can be incorporated into an IA questionnaire. Young presented an eight-item IA diagnostic questionnaire that included $\mathrm{S}_{\mathrm{CS}}, \mathrm{S}_{\mathrm{DC}}, \mathrm{S}_{\mathrm{I}} / \mathrm{S}_{\mathrm{SO}}, \mathrm{S}_{\mathrm{MM}}, \mathrm{S}_{\mathrm{PS}}, \mathrm{S}_{\mathrm{TM}}, \mathrm{S}_{\mathrm{T}}$, and $\mathrm{S}_{\mathrm{WS}}$ [2]. Griffiths proposed that $\mathrm{S}_{\mathrm{I}} / \mathrm{S}_{\mathrm{H}}, \mathrm{S}_{\mathrm{MM}}, \mathrm{S}_{\mathrm{CS}}, \mathrm{S}_{\mathrm{PS}}, \mathrm{S}_{\mathrm{T}}$, and $\mathrm{S}_{\mathrm{Ws}}$ were the core components of addiction [32]. The IGD questionnaire comprises $\mathrm{S}_{\mathrm{A}}, \mathrm{S}_{\mathrm{CS}}, \mathrm{S}_{\mathrm{DC}}, \mathrm{S}_{\mathrm{I}}$, $\mathrm{S}_{\mathrm{MM}}, \mathrm{S}_{\mathrm{PS}}, \mathrm{S}_{\mathrm{SO}}, \mathrm{S}_{\mathrm{T}}$, and $\mathrm{S}_{\mathrm{WS}}$ [14], and the CIAS consists of $\mathrm{S}_{\mathrm{CS}}, \mathrm{S}_{\mathrm{I}} / \mathrm{S}_{\mathrm{H}}$, $\mathrm{S}_{\mathrm{T}}, \mathrm{S}_{\mathrm{TM}}$, and $\mathrm{S}_{\mathrm{Ws}}$ [3]. Authors suggested that $\mathrm{S}_{\mathrm{I}} / \mathrm{S}_{\mathrm{H}}, \mathrm{S}_{\mathrm{MM}}, \mathrm{S}_{\mathrm{CS}}, \mathrm{S}_{\mathrm{SO}}$, $\mathrm{S}_{\mathrm{T}}, \mathrm{S}_{\mathrm{PS}}$, and $\mathrm{S}_{\mathrm{Ws}}$ be used to design a questionnaire [5]. Authors structured their IA questionnaire by using the psychological symptoms of $\mathrm{S}_{\mathrm{A}}, \mathrm{S}_{\mathrm{C}}, \mathrm{S}_{\mathrm{CS}}, \mathrm{S}_{\mathrm{DC}}, \mathrm{S}_{\mathrm{H}} / \mathrm{S}_{\mathrm{SO}}, \mathrm{S}_{\mathrm{I}}, \mathrm{S}_{\mathrm{MM}}, \mathrm{S}_{\mathrm{PS}}, \mathrm{S}_{\mathrm{T}}$, and $\mathrm{S}_{\mathrm{Ws}}$ [9].

IA questionnaires are typically translated into various languages or modified into suitable structures for application in different countries or areas. For example, Young's IA Test is a well-known questionnaire for assessing IA risk [15]. Researchers in Germany converted this 20-item test into a 12-item short-form version [16]. Researchers also modified the nine IGD criteria outlined in the DSM, Fifth Edition, into a new nine-item short version for UK Internet users [17]. The CIAS is a common Chinese-language tool for evaluating IA symptoms [3]. Researchers in Hong Kong reduced the 26 items of the CIAS to 19 [18]. The aforementioned studies have adopted factor analysis to extract the psychological symptoms of IA and confirm the reliability and validity of their new IA questionnaire versions [1618]. However, the factor analysis employed for extracting these symptoms used single-dimension calculations, and thus may be unable to analyze non-Markovian problems. Non-Markovian indicates that the optimum decision cannot be determined only from the current state, and memory is needed to assist decisionmaking. In addition, current IA questionnaires are limited to assessing prolonged Internet use (over 6 months) after browsing Internet (also called narrative surveys). Few questionnaires 
evaluate the current experiences of the Internet user during browsing Internet (also termed the experience sampling method). Therefore, the design of an IA questionnaire for the immediate detection of IA risk is a substantial challenge.

Table 1: Psychological symptoms of IA

\begin{tabular}{|c|c|}
\hline Sympto & Explanation \\
\hline $\mathrm{S}_{\mathrm{A}}$ & $\begin{array}{l}\text { stop or reduce take part in other activities due to using } \\
\text { the Internet }\end{array}$ \\
\hline $\mathrm{S}_{\mathrm{C}}$ & desire to use the Internet \\
\hline $\mathrm{S}_{\mathrm{CS}}$ & $\begin{array}{l}\text { try to stop or reduce Internet use repeatedly without } \\
\text { success }\end{array}$ \\
\hline $\mathrm{S}_{\mathrm{DC}}$ & lie about their amount of using the Internet \\
\hline $\mathrm{S}_{\mathrm{H}}$ & $\begin{array}{l}\text { produce psychosocial and physical problems due to } \\
\text { overusing the Internet }\end{array}$ \\
\hline $\mathrm{S}_{\mathrm{I}}$ & lose relationships due to overusing the Internet \\
\hline $\mathrm{S}_{\mathrm{MM}}$ & $\begin{array}{l}\text { use the Internet to escape from stress or relieve } \\
\text { negative mood }\end{array}$ \\
\hline $\mathrm{S}_{\mathrm{PS}}$ & $\begin{array}{l}\text { always think about Internet activities or expect the } \\
\text { next Internet activities }\end{array}$ \\
\hline $\mathrm{S}_{\mathrm{SO}}$ & $\begin{array}{l}\text { risk the educations or occupations due to overusing the } \\
\text { Internet }\end{array}$ \\
\hline $\mathrm{S}_{\mathrm{T}}$ & $\begin{array}{l}\text { need to increase time spent on the Internet to achieve } \\
\text { the same level of satisfaction as before }\end{array}$ \\
\hline $\mathrm{S}_{\mathrm{TM}}$ & reduce sleep and other activities to use the Internet \\
\hline $\mathrm{S}_{\mathrm{WS}}$ & feel displeasure when stop or reduce using the Internet \\
\hline
\end{tabular}

\subsection{Physiological response of IA}

Individuals with IA experience dynamic emotional stimulation on the Internet and then regulate psychophysiological responses instantaneously. Cardiovascular, respiratory, body temperature, skin conductance, and other responses in IA have been observed for short periods. The aim of such monitoring is to provide additional data that can be analyzed with IA questionnaires. Relevant results indicated that the risk of IA was positively associated with respiratory responses during 6 min of Internet browsing but negatively related to peripheral temperature [19]. Compared with individuals with low-risk Internet addiction (LIA), individuals with high-risk Internet addiction (HIA) showed higher skin conductance responses after $15 \mathrm{~min}$ of Internet use [10]. The respiratory sinus arrhythmia responses of users with HIA while watching negative and positive videos of 2 to 3 min were lower than that of users with LIA [20]. The heart rate and systolic blood pressure of individuals with problematic Internet use both increased after 2 min of stopping Internet use [21]. When playing an online game for $5 \mathrm{~min}$, the high-frequency heart rate variability and cardiopulmonary synchrony for problematic Internet users in an excessive online gaming group were lower than those of the healthy group [22]. Compared with 5 min of baseline values, the natural logarithm of high-frequency heart rate variability of gamers with IGD was lower during 5 min of online gaming with attention [23].

Among the physiological signals, respiration is the only one that reflects emotional responses and affects emotions, motivation, and other physiological signals. Patients with IA and anxiety disorder obtained alleviation for their addiction and anxiety symptoms after 10 weeks of cognitive-behavioral therapy with breathing exercises [33]. Mindful breathing has also been employed to assist gamers with IGD [34] and individuals with a gambling addiction [35] to relieve their desire to play. For physiological regulation, respiration is related to the vagus nerve, which modulates sympathetic and parasympathetic nervous system activities. Slow respiratory rates reduce sympathetic nervous activity or increase parasympathetic nervous activity. Inhalation and exhalation change the intrathoracic pressure, which in turn affects the heart rate and blood pressure. Individuals with high-risk IGD reduced their respiratory rates by using slow and deep breathing exercises while watching negative films [31]. Therefore, the respiration response is a key feature for detecting IA risk, and respiratory adjustments constitute a valuable means of affecting psychophysiological responses.

The major IA-related features are generally extracted from physiological signals using descriptive statistics and generic mathematics methods. However, these methods are limited in analyzing physiological signals with dynamic and instantaneous changes and solving non-Markovian problems. The extraction of instantaneous physiological features and investigation of the relationship between each instantaneous physiological component are crucial for determining IA.

\subsection{IA detection using machine learning}

Some researchers have proposed machine learning models as computer-aided systems for detecting IA and extracting IA-related psychological and physiological features. Impulsive and compulsive symptoms were adopted to construct prediction models of problematic Internet use by employing naïve Bayes, random forest, and logistic regression methods [24]. Authors used a decision tree to determine IGD and extract the features of gaming cost, gaming time on weekdays and weekends, and offline gaming social, marriage, and self-perceptions [25]. In supervised machine learning, demographic information, gamer statistics, and selfesteem scores were input to classify IGD [26]. Authors combined long short-term memory with an adversarial autoencoder to predict the gamer's IGD risk using duration, costs, feelings of depressive responses, and contextual information in games as input data [27]. Social data from Facebook and Instagram were applied to detect problematic social network use by employing a support vector machine framework [7]. The aforementioned studies were required to collect data over a long period of time. Authors proposed that 10- or 25-s biosignals, such as heart and respiratory rates, eye blinks, and saccadic movements, be input as variables into the support vector machine to determine the urge of an IGD group for gaming [28]. Although long-term and short-term information has been used to predict addiction, the lack of algorithms impedes the use of instantaneous physiological response measurements in IA detection.

\section{Research Methodology}

\subsection{Empirical mode decomposition}

Empirical mode decomposition (EMD) [36] is an adaptive filter method applied to decompose nonlinear and nonstationary signals into oscillatory components from high to low frequencies; these components are also termed intrinsic mode functions (IMFs). The decomposition process of EMD is expressed in Algorithm 1. 


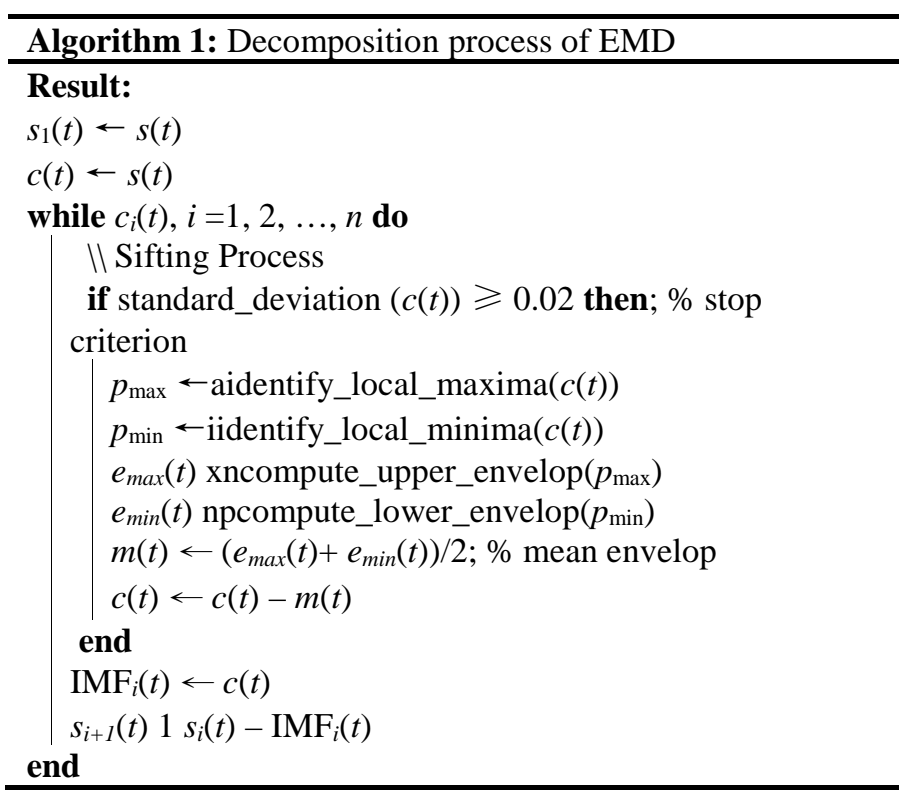

EMD, however, has boundary-effect and mode-mixing problems. Boundary-effect indicates IMF endpoint distortions. Mode mixing refers to different oscillatory waveforms being present in a single IMF. These two problems lead to difficult or erroneous interpretation of the physical meaning of the IMFs but can be resolved using the EEMD method [37]. The analysis process of EEMD involves the addition of Gaussian noise to the source signal; this noise-added signal is then decomposed using the sifting process of the EMD method.

\section{2. $X C S$}

The XCS is a powerful machine learning model with high interpretability and flexibility. The interpretability of the XCS stems from the use of a set of collaborative rules known as a population set $[\mathrm{P}]$ to express the solution of the problem domain. The interpretation of each rule is the "IF condition THEN action," with the condition part of each rule corresponding to the detected environmental state and coded with a ternary character $\{0,1, \#\}$. The \# symbol is termed "don't care," which indicates that the corresponding environmental state can be ignored regardless of whether it is 0 or 1 . The action part of each rule is the decisionmaking that the XCS executes. If the output is a binary classification problem, the action can be encoded as a binary string $\{0,1\}$. Furthermore, each rule contains the following three parameters to indicate the prediction and quality of each rule: 1 ) prediction, which is used to predict how much feedback can be obtained from the environment after the action is executed; 2) prediction error, which is used to calculate the error between the prediction and actual feedback; and 3) fitness, which is used to evaluate the accuracy and quality of the prediction. A modular architecture allows XCS to address many problem areas flexibly, including performance components, exploration components, and credit assignment.

The performance component is responsible for detecting the state of the environment and making decisions. First, the XCS converts the detected environmental state into a binary string. Second, the condition part of each rule in the $[\mathrm{P}]$ is compared with the environmental state, and the rule is moved to the matching set $[\mathrm{M}]$ only if the conditions of the rule match the environmental state.
If $[\mathrm{M}]$ is empty, the XCS initiates the covering mechanism to generate new rules that satisfy the environmental state. Third, the XCS must select an action to execute according to the rules in $[\mathrm{M}]$. The system uses rules with the same action in $[\mathrm{M}]$ to calculate the expected feedback of the action and form a prediction array [PA]. The XCS adopts two mechanisms, exploration and exploitation, to select the action. In the exploration mode, the expected feedback calculated by each action in [PA] is used as the probability of random selection; in the exploitation mode, the system directly selects the action with the greatest expected feedback. Fourth, the selected action is executed through the effector, and the rules in [M] that match the action move to the action set [A] to update the parameters. The XCS repeats these four steps until convergence or the maximum number of iterations has been reached.

The exploration components are used to assist the XCS in generating new rules to search for the optimum solution in the problem domain. Evolutionary computation (EC) introduces small random factors to gradually optimize the solution and is suitable for the exploration components of the XCS. Typically, the XCS adopts the genetic algorithm (GA) in EC as its exploration components. GA solves the optimization problem by simulating natural selection, heredity, crossover, and mutation mechanisms in evolutionary biology. In the XCS, the GA randomly selects two rules from [P] for inheritance based on the fitness of each rule as the probability. Subsequently, the GA performs crossover on each part of the condition in accordance with the two rules based on the probability $\chi$ and mutates each part with probability $\mu$. The GA generates two new rules to replace the rules with lower fitness in [P] to assist the XCS in identifying the global optimum in the problem domain.

The credit assignment assigns and updates the parameters of the rules in [A]. The XCS uses the feedback from the environment to modify the prediction of each rule in [A] as a reference parameter for the next iteration. The modified technique uses the Widrow-Hoff delta rule, as described in Eq. (1), where $p$ is the prediction of the rule, $P$ is the actual feedback obtained from the environment, $t$ is the current iteration number, and $\beta$ is the learning rate $(0<\beta \leq 1)$. The XCS can then use the absolute error to correct the prediction error of the rule, as detailed in Eq. (2), where $\varepsilon$ denotes the prediction error. The last parameter fitness is used to evaluate the quality of the rule. The calculation of fitness is based on the relative accuracy of the rule in [A]. First, the XCS must calculate the accuracy $k_{t}$ of each rule in [A], as expressed in Eq. (3), where $\alpha$ controls the attenuation of the exponential function (0 $<\alpha<1), \varepsilon_{t}$ is the prediction error of the rule at time $t$, and $\varepsilon_{0}$ is the tolerable error. Subsequently, the XCS calculates the relative accuracy $k_{t}^{\prime}$ of the rule compared with the other rules in [A], as described in Eq. (4). The system utilizes the relative accuracy $k_{t}$ ' as the fitness of the rule and employs the Widrow-Hoff delta rule to update, as detailed in Eq. (5), where $F$ is the fitness of the rule.

$$
\begin{gathered}
p_{t+1}=p_{t}+\beta\left(P_{t}-p_{t}\right) \\
\varepsilon_{t+1}=\varepsilon_{t+1}+\beta\left(\left|P_{t}-p_{t}\right|-\varepsilon_{t}\right) \\
k_{t}=\left\{\begin{aligned}
\exp \left[\frac{(\ln \alpha)\left(\varepsilon_{t}-\varepsilon_{0}\right)}{\varepsilon_{0}}\right], & \varepsilon_{t}>\varepsilon_{0} \\
1, & \text { otherwise }
\end{aligned}\right.
\end{gathered}
$$




$$
\begin{gathered}
k_{t}{ }^{-}=\frac{k_{t}}{\sum_{k_{t} \in[A]} k_{t}} \\
F_{t+1}=F_{t}+\beta\left(k_{t}^{\prime}-F_{t}\right)
\end{gathered}
$$

The XCS provides the rule with a numerosity parameter to record how many of the same rules are in [P] and uses a rule as a representative of these rules. Therefore, the same rules must only be matched once to maximize the computation speed. The subsumption mechanism is also added to assist in generating the most accurate and general rules. In the subsumption mechanism, one rule can subsume another rule, and the rule therefore undergoes a certain amount of training that strengthens its generalizability and accuracy. If rule $r_{a}$ can subsume another rule $r_{b}, r_{b}$ is removed from [P], and the numerosity of $r_{a}$ increases by one. The subsumption mechanism usually acts on the GA and [A].

\section{3. $X C S R$}

The encoding method used in the condition of the rule can only process discrete variables. Wilson proposed to modify the ternaryalphabet encoding of the condition into interval predicates to enable the XCS to process continuous variables [30]. The expression of interval predicates is $\operatorname{int}_{i}=\left(c_{i}, s_{i}\right)$, where int $t_{i}$ is the rule condition that the $i$ th interval predicate corresponds to the $i$ th variable, $c_{i}$ is the central value of the range, and $s_{i}$ refers to the size of the coverage boundary. Changing the expression of the condition modifies the mechanism of components, such as matching, covering, and the GA. For matching, assuming the ith variable is $x_{i}$, if $c_{i}-s_{i} \leq x_{i}<c_{i}+s_{i}$, then the int $t_{i}$ matches $x_{i}$. For covering, the XCS generates a rule whose interval predicates cover all input variables. The generation method is to set $c_{i}$ to $x_{i}$, and $s_{i}$ is set to a random value between 0 and $s_{r}$, where $s_{r}$ is defined by the user. For the GA, crossover exchanges the int $t_{i}$ with probability $\chi$; mutation adds a random value from 0 to $s_{m}$ to the range of int $t_{i}$ with probability $\mu$, where $s_{m}$ is defined by user. The remaining mechanism is the same as the XCS operation, with this modified version known as the XCSR. The modular structure allows the $\mathrm{XCS}$ to be flexibly modified to adapt to various problem domains.

\section{Experiment}

\subsection{Design of experiment}

\section{(1) Objective of this experiment}

The CIAS scores of participants with HIA and LIA were collected, and their respiratory signals were observed while they watched online game videos. This study was granted ethical approval by National Chiao Tung University (NCTU) Research Ethics Committee for Human Subject Protection (Approval Number: NCTU-REC-102-009-e).

\section{(2) Method}

To assess the risk of IA, we adopted the CIAS, which contains 26 items, with each item ranked from 1 (strong disagreement) to 4 (strong agreement) [3]. The total score of the CIAS ranged from 26 to 104, with a score of 26 to 63 and 64 to 104 reflecting LIA and HIA, respectively. This study also adopted the IGD questionnaire to evaluate IGD risk; this questionnaire consists of nine items requiring yes or no responses [14]. The IGD questionnaire score ranged from 0 to 9 , with 0 to 4 and 5 to 9 representing non-IGD and IGD, respectively. The internal consistency of the CIAS and IGD questionnaires, as estimated using Cronbach's $\alpha$, was 0.94 and 0.81 , respectively. The extent of participants' experience using computers and the Internet were recorded, and the Self-Assessment Manikin (SAM) was adopted to evaluate the emotions of participants. The SAM measures emotional valence and arousal on a 9-point Likert scale. A 5-point score on valence represents neutral emotion, and anything higher indicates positive emotions. Conversely, a score lower than 5 is indicative of negative emotions. Arousal was scored from 1 (no feeling) to 9 (strong feeling). To arouse a craving for gaming, 2 min of online game videos were selected from League of Legends (LOL; Riot Games, Los Angeles, CA, USA), MapleStory (MS; Nexon, Seoul, South Korea), and Resident Evil (RE; Capcom, Osaka, Japan). Compared with the active stimuli of playing games, watching clips as passive stimuli may induce more physiological stability, potentially lowering the difference in psychological responses between participants.

\section{(3) Indices}

The 26-item scores of CIAS and instantaneous frequency (IF) of respiratory signal were used to detect the risk of IA.

\section{(4) Participant}

Participants aged 20-40 years and with no symptoms of depression and anxiety, and cardiovascular diseases were included in the study. Each participant provided written informed consent. A total of 50 participants (36 men and 14 women) were recruited from NCTU.

\section{(5) Procedure}

Each participant stared at a gray picture to familiarize themselves with the environment and relax physically at the beginning of the experiment. To induce a feeling of calm, the participant looked at a gray picture for $2 \mathrm{~min}$ before each video was played. For emotional stimuli, participants watched three videos (LOL, MS, and RE) in a random order. After each video, participants completed the SAM questionnaire, after which time the gray picture was displayed for another 2 min to allow the participant to recover from emotional arousal. During the experimental procedure, respiratory inductance plethysmography (RIPmate Inductance Belt, Ambu, Ballerup, Denmark) and electrocardiography (Ag/AgCl spot electrodes attached to the left and right subclavian and left lumber regions) were used to measure abdominal wall movement and electrocardiography signals, respectively. These signals were acquired through a DAQCard (USB 6218, NI, Austin, TX, USA) with a $1000 \mathrm{~Hz}$ sampling rate. To investigate instantaneous respiratory responses, this study only analyzed abdominal wall movement signals.

\subsection{Data analysis}

\section{(1) Method}

The XCSR model was used to detect the risk of IA by using the CIAS data and the IF of respiration as the input features (Figure 1). The abdominal movement signal was analyzed as the IF according to the signal processing step described in a previous study [36], including integral calculation, signal baseline correction, downsampling, signal decomposition, and transformation. 


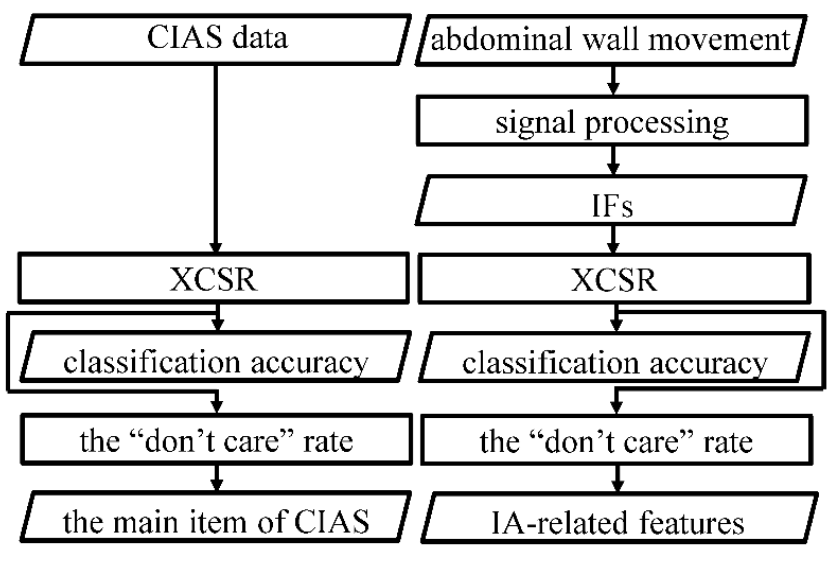

Figure 1: Data analysis procedure.

For statistical calculation, the Mann-Whitney $U$ test was performed to compare differences in the questionnaire data and average IF between the HIA with IGD and LIA with non-IGD groups. The statistical significance was set as $p<0.05$. To assess the performance of the average IF of respiration in discriminating between HIA with IGD and LIA with non-IGD, the receiver operating characteristic curve (ROC) was calculated, and the area under the curve (AUC) of the ROC indicated the predictive accuracy. The AUC ranged from zero (reflecting incorrect prediction) to one (representing correct prediction); the AUC was 0.7 , indicating acceptable predictive power.

\section{(2) Procedure}

The integral calculation converted the abdominal movement signal into the volume change of the abdomen by using Simpson's rule. The volume changes of the abdomen were then used to perform baseline correction using the linear fit. To investigate the frequency band of respiratory muscle contractions, the corrected signal was downsampled from a rate of 1000 to $50 \mathrm{~Hz}$. Subsequently, the EEMD method was used to decompose the downsampled signal into eight IMFs. With reference to the frequency bandwidth [38], IMF $-\mathrm{IMF}_{2}, \mathrm{IMF}_{3}-\mathrm{IMF}_{4}, \mathrm{IMF}_{5}-\mathrm{IMF}_{6}$, and $\mathrm{IMF}_{7}-\mathrm{IMF}_{8}$ represented the diaphragm, respiratory muscle cluster on the abdomen, respiratory wall movement on the abdomen, and body movement components, respectively. In the transformation step, each IMF was transformed into IF by using the normalized direct quadrature [39]. The average value of IF during the viewing of the LOL, MS, and RE videos was treated as the probability of crossover operation $(\chi)=0.8$, the probability of the instantaneous psychological features that were input into the XCSR model.

Parameters in the XCSR were set as follows [29, 30, 40]: the population size $(N)$ constituted 350 classifiers, probability of \# $(\mathrm{P \#})$ $=0.33$, initial rule $\varepsilon=0$, initial rule $p=10$, initial rule $F=10$, learning rate $(\beta)=0.2$, accuracy function $\left(\varepsilon_{0}\right)=10, v=5$, $\varepsilon_{0}=10$, $\alpha=0.1, \phi=0.1$, mutation operation $(\mu)=0.04$, threshold parameter of the GA $=50$, deletion thresholds of the $\mathrm{GA}=20$, and subsumption thresholds of the GA $=20$; moreover, the reward setup for accurate prediction of HIA with IGD and LIA with nonIGD was set as 1000 , or otherwise 0 . The iteration number of the XCSR method was 1950 for both the 26-item score in the CIAS and 24 IF values of respiration as input features. The moving average per 50 exploitations was used to calculate the classification accuracy of the XCSR. To verify the items in the CIAS and extract the crucial instantaneous respiratory components for detecting the IA risk, rules with a reward of 1000 in [P] were selected, and then the rate of non-“don't care” values ("non-\#”) in these rules was calculated. To effectively evaluate the training quality of the XCSR and avoid bias, 10-fold cross-validation was used. To eliminate the unevenness of the IF at different frequency bandwidths, the IF underwent min-max normalization. The XCSR does not update any parameters nor generate new rules for the testing data and only uses the exploitation mode when selecting and performing actions on this data. All data management, respiratory signal processing, and XCSR execution operation were conducted using LabVIEW (v.2021, NI, Austin, TX, USA).

\section{Results}

Of all participants, 34\% were from the College of Electrical and Computer Engineering, 28\% from the College of Computer Science, $14 \%$ from the College of Management, $8 \%$ from the College of Humanities and Social Sciences, 6\% from the College of Hakka Studies, 6\% from the College of Engineering, and 4\% were from other departments. Participants were stratified into four groups according to their CIAS and IGD questionnaire scores, namely the LIA with non-IGD, LIA with IGD, HIA with non-IGD, and HIA with IGD groups. This study focused on investigating the psychological symptoms and physiological responses of the LIA with non-IGD and HIA with IGD groups only. Table 2 lists the demographic information and questionnaire responses of the two groups. No significant differences in age, computer and Internet use experience, or emotional valence and arousal were observed between the two groups. The total scores of the CIAS and IGD questionnaires of the HIA with IGD group were significantly higher than those of the LIA with non-IGD group. The emotional valence results indicated that the LOL and MS videos evoked positive emotions, whereas the RE video prompted negative emotions.

Table 2: Demographic information and questionnaire responses

\begin{tabular}{|c|c|c|}
\hline & $\begin{array}{l}\text { LIA with } \\
\text { non-IGD }\end{array}$ & $\begin{array}{c}\text { HIGD with } \\
\text { IGD }\end{array}$ \\
\hline Gender (men, women) & 12,9 & 14,4 \\
\hline Age (years old) & $22.62 \pm 1.79$ & $23.67 \pm 5.27$ \\
\hline Computer experience (year) & $13.19 \pm 2.71$ & $12.44 \pm 4.50$ \\
\hline Internet experience (year) & $11.71 \pm 2.63$ & $10.89 \pm 4.19$ \\
\hline CIAS & $52.10 \pm 8.03$ & $79.33 \pm 8.88^{* *}$ \\
\hline IGD questionnaire & $1.48 \pm 1.25$ & $6.58 \pm 1.54^{* *}$ \\
\hline valence_LOL & $5.95 \pm 1.32$ & $6.61 \pm 1.33$ \\
\hline arousal_LOL & $4.33 \pm 2.01$ & $5.28 \pm 1.53$ \\
\hline valence_MS & $5.76 \pm 1.79$ & $5.56 \pm 1.46$ \\
\hline arousal_MS & $4.33 \pm 1.88$ & $4.22 \pm 1.73$ \\
\hline valence_RE & $3.90 \pm 1.87$ & $4.39 \pm 1.61$ \\
\hline arousal_RE & $6.14 \pm 1.80$ & $5.78 \pm 1.63$ \\
\hline
\end{tabular}

** $\mathrm{p}<0.01$ in the Mann-Whitney U test

The results of classification accuracy of the XCSR with an average of 30 repetitions using 26-item CIAS scores and 24 IFs of respiration in response to online video stimuli are illustrated in Figure 2 and Figure 3, respectively. The classification accuracy of the XCSR model was over 95\%. Table 3 presents the mean \pm standard deviation scores of each item in the CIAS for the two 
groups and the "non-\#" rate of each item. The score of each item for the HIA with IGD group was significantly higher than that of the LIA with non-IGD group. The "non-\#” rate of the 11th, 14th, 19th, and 22nd items in $\mathrm{S}_{\mathrm{CS}}$; 12th, 13th, 15th, 17th, 18th, and 21st items in $\mathrm{S}_{\mathrm{I}} / \mathrm{S}_{\mathrm{H}}$; 6th item in $\mathrm{S}_{\mathrm{T}}$; 1st, 8th, 23rd, and 25th items in $\mathrm{S}_{\mathrm{TM}}$; and 2nd, 4th, 10th, and 16th items in $\mathrm{S}_{\mathrm{w}}$ were higher than the 0.50 value for the CIAS. The 10-fold cross-validation of the XCSR using the 26-item CIAS score and 24 IFs of respiration in response to online video stimuli is described in Table 4 and Table 5, respectively; the average accuracy of 10 -fold cross-validation for the XCSR was $87 \%$ and $78 \%$, respectively. Table 6 presents the mean \pm standard deviation of the IFs of respiration of the two groups while watching the LOL, MS, and RE videos as well as the "non-\#" rate of each IF of respiration. The $\mathrm{IF}_{2}$ of the HIA with IGD group while watching the RE video stimuli was significantly higher than that of the LIA with non-IGD group. Except for $\mathrm{IF}_{5}$ during the MS video and $\mathrm{IF}_{5}$ and $\mathrm{IF}_{7}$ during the RE video, the "non\#" rate of all IFs was higher than the 0.50 value. The ability to predict the risk of IA using IF values, as calculated with the AUC of the ROC, is detailed in Table 7.

\section{Discussion}

In this study, the XCSR method as a rule-based machine learning system was used to aid in detecting the risk of IA. The "don't care" attribute of the XCSR was employed to verify the items of the CIAS and extract the instantaneous respiratory features for the classification of IA. The results demonstrated that the XCSR model could identify HIA with IGD group or LIA with non-IGD group with a classification accuracy of over $95 \%$. The average accuracy of the 10 -fold cross-validation was also over 78\%. Researchers have proposed that self-esteem scores [26], impulsive and compulsive symptoms [24], and physiological signals [28] can serve as the input variables of machine learning systems to predict behavioral addictions. Our findings suggested that the XCSR model is an appropriate computer-aided tool for detecting IA. The psychological symptoms in the CIAS and instantaneous respiratory responses are vital indexes for assessing risky Internet use.

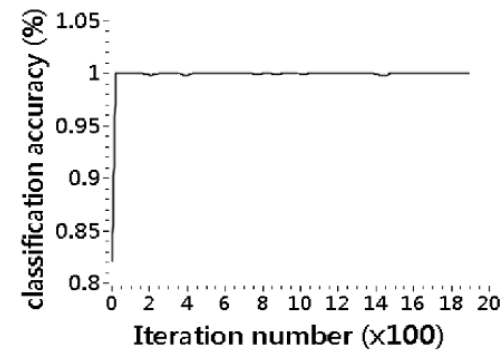

Figure 2: Classification accuracy of the XCSR with the CIAS data as the input feature.

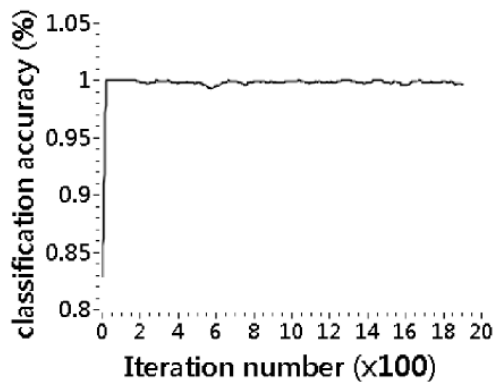

Figure 3: Classification accuracy of the XCSR with the IF of respiration data as the input feature.

Table 3: Psychological symptoms in the CIAS and their corresponding items (modified from [1])

\begin{tabular}{|c|c|c|c|c|c|}
\hline \multicolumn{2}{|c|}{ Symptom } & \multirow{2}{*}{\begin{tabular}{|l|} 
item \\
I unable control my impulse of Internet use \\
\end{tabular}} & \multirow{2}{*}{$\begin{array}{c}\begin{array}{c}\text { LIA with } \\
\text { non-IGD }\end{array} \\
1.81 \pm 0.68\end{array}$} & \multirow{2}{*}{$\begin{array}{c}\begin{array}{c}\text { HIA with } \\
\text { IGD }\end{array} \\
2.89 \pm 0.74^{* *}\end{array}$} & \multirow{2}{*}{$\begin{array}{c}\begin{array}{r}\text { not \# } \\
\text { rate }\end{array} \\
0.58 \\
\end{array}$} \\
\hline $\mathrm{S}_{\mathrm{CS}}$ & & & & & \\
\hline & & When waking up every morning, my first thought is to use the Internet & $2.00 \pm 0.89$ & $3.05 \pm 0.78^{* *}$ & 0.75 \\
\hline & & After stopping Internet use, I crave it again & $2.33 \pm 0.73$ & $3.32 \pm 0.58^{* *}$ & 0.67 \\
\hline & & Without the Internet, my life would be joyless & $1.76 \pm 0.77$ & $2.79 \pm 0.85^{* *}$ & 0.42 \\
\hline & & I try to spend less time on the Internet, but failure & $2.14 \pm 0.57$ & $3.05 \pm 0.71^{* *}$ & 0.67 \\
\hline \multirow[t]{7}{*}{$\mathrm{S}_{\mathrm{I}} / \mathrm{S}_{\mathrm{H}}$} & & Even if the Internet negatively affects my interpersonal relationships, my use remains unreduced & $1.43 \pm 0.60$ & $3.21 \pm 0.71^{* *}$ & 0.42 \\
\hline & & I find that I reduce the time spent with friends due to the Internet & $1.81 \pm 0.51$ & $2.95 \pm 0.78^{* *}$ & 0.67 \\
\hline & & I feel aches and soreness in the back or other discomfort due to Internet use & $2.00 \pm 0.84$ & $3.11 \pm 0.94^{* *}$ & 0.92 \\
\hline & & Using Internet has negative effects on my education or work & $1.86 \pm 0.73$ & $2.84 \pm 0.60^{* *}$ & 0.75 \\
\hline & & I reduced interaction with my family due to Internet use & $1.76 \pm 0.77$ & $3.05 \pm 0.85^{* *}$ & 0.58 \\
\hline & & I reduce my recreational activities to use the Internet & $2.00 \pm 0.63$ & $3.16 \pm 0.83^{* *}$ & 0.67 \\
\hline & & Using the Internet has negative effects on my health & $2.10 \pm 0.70$ & $2.95 \pm 0.78^{* *}$ & 0.83 \\
\hline \multirow[t]{4}{*}{$\mathrm{S}_{\mathrm{T}}$} & & I perceive my Internet use as getting longer and longer & $2.43 \pm 0.51$ & $3.05 \pm 0.71^{* *}$ & 0.50 \\
\hline & & I spend more time on the Internet than I originally intended & $2.71 \pm 0.64$ & $3.53 \pm 0.51^{* *}$ & 0.58 \\
\hline & & I have spent more time on the Internet since last semester & $2.00 \pm 0.63$ & $2.95 \pm 0.71^{* *}$ & 0.33 \\
\hline & & I need to increase the amount of time I use the Internet to achieve the same satisfaction as before & $1.67 \pm 0.48$ & $2.63 \pm 0.83^{* *}$ & 0.42 \\
\hline \multirow[t]{5}{*}{$\mathrm{S}_{\mathrm{TM}}$} & & I have been told more than once that I spend too much time on the Internet & $2.48 \pm 0.75$ & $3.21 \pm 0.79^{* *}$ & 0.58 \\
\hline & & My sleep time is less than 4 hours from using the Internet & $1.76 \pm 0.89$ & $3.16 \pm 0.96^{* *}$ & 0.67 \\
\hline & & I am used to reducing my sleep time to use the Internet & $1.52 \pm 0.75$ & $2.79 \pm 0.79^{* *}$ & 0.67 \\
\hline & & I do not eat on time due to using the Internet & $2.05 \pm 0.80$ & $2.84 \pm 0.90^{* *}$ & 0.58 \\
\hline & & I use the Internet all night, which causes daytime tiredness & $2.33 \pm 1.02$ & $3.00 \pm 0.58^{* *}$ & 0.50 \\
\hline \multirow[t]{5}{*}{ Sws } & & I feel displeasure when I stop using the Internet for a period of time & $2.05 \pm 0.67$ & $3.26 \pm 0.73^{* *}$ & 0.58 \\
\hline & & I feel restless and irritable when the Internet is unavailable & $2.52 \pm 0.87$ & $3.37 \pm 0.68^{* *}$ & 0.75 \\
\hline & & I feel energetic upon using the Internet regardless of a fatiguing experience & $1.62 \pm 0.59$ & $2.84 \pm 0.76^{* *}$ & 0.50 \\
\hline & & I feel distressed when stopping the Internet use for a period of time & $1.57 \pm 0.51$ & $2.89 \pm 0.74^{* *}$ & 0.83 \\
\hline & & I feel like I am missing something when stopping the Internet use for a period of time & $2.38 \pm 0.97$ & $3.37 \pm 0.60^{*}$ & 0.75 \\
\hline
\end{tabular}


The results indicated that the 11th, 14th, 19th, and 22nd items in $\mathrm{S}_{\mathrm{CS}}$; 12th, 13th, 15th, 17th, 18th, and 21st items in $\mathrm{S}_{\mathrm{I}} / \mathrm{S}_{\mathrm{H}}$; 6th item in $\mathrm{S}_{\mathrm{T}}$; 1st, 8th, 23rd, and 25th items in $\mathrm{S}_{\mathrm{TM}}$; and 2nd, 4th, 10th, and 16th items in $\mathrm{S}_{\mathrm{w}}$ were maintained in the CIAS item verification. This result, however, is inconsistent with that of other works. Authors modified the items in the CIAS for grade 7-to-13 Hong Kong students, retaining the 14th and 20th items in $\mathrm{S}_{\mathrm{CS}}$; 12th, 13th, 15th, 17th, 18th, and 21st items in $\mathrm{S}_{\mathrm{I}} / \mathrm{S}_{\mathrm{H}}$; 3rd, 6th, 9th, and 24th items in $\mathrm{S}_{\mathrm{T}}$; 1st, 8th, 23rd, and 26th items in $\mathrm{S}_{\mathrm{TM}}$; and 2nd, 5th, and 16th items in $S_{W}$ [18]. Authors changed the 26-item CIAS into a 10-item smartphone addiction inventory, retaining the 22nd item in $\mathrm{S}_{\mathrm{CS}}$; 7th, 13th, and 15th items in $\mathrm{S}_{\mathrm{I}} / \mathrm{S}_{\mathrm{H}}$; 3rd, 6th, and 9th items in $\mathrm{S}_{\mathrm{T}}$; 23rd item in $\mathrm{S}_{\mathrm{TM}}$; and 2nd and 4th items in $\mathrm{S}_{\mathrm{W}}$ for Taiwan students aged 18 to 31 years [41]. These inconsistent results are likely attributable to differences in age, country, analysis method, or assessment of psychological symptoms between studies. Our findings must be validated with more data.

In this study, the "don't care" rate of $\mathrm{IF}_{1}-\mathrm{IF}_{8}$ of respiration during LOL video stimuli; $\mathrm{IF}_{1}-\mathrm{IF}_{4}$ and $\mathrm{IF}_{6}-\mathrm{IF}_{8}$ of respiration during MS video stimuli; and $\mathrm{IF}_{1}-\mathrm{IF}_{4}, \mathrm{IF}_{6}$, and $\mathrm{IF}_{8}$ of respiration during RE video stimuli were lower than $0.50 . \mathrm{IMF}_{1}-\mathrm{IMF}_{2}, \mathrm{IMF}_{3}-$ $\mathrm{IMF}_{4}, \mathrm{IMF}_{5}-\mathrm{IMF}_{6}$, and $\mathrm{IMF}_{7}-\mathrm{IMF}_{8}$ represented the diaphragm, respiratory muscle cluster on the abdomen, respiratory wall movement on the abdomen, and body movement components, respectively [38]. The LOL and MS videos aroused positive emotions in the participants, whereas the RE video evoked negative emotions.

Table 4: The 10-fold cross-validation of the XCSR with the CIAS data as the input feature

\begin{tabular}{cccccc}
\hline Testing & 1 & 2 & 3 & 4 & 5 \\
\hline Accuracy & 1.00 & 1.00 & 1.00 & 0.50 & 1.00 \\
\hline Testing & 6 & 7 & 8 & 9 & 10 \\
\hline Accuracy & 1.00 & 0.75 & 0.75 & 1.00 & 0.67 \\
\hline Average accuracy & & & 0.87 & & \\
\hline
\end{tabular}

Table 5: The 10-fold cross-validation of the XCSR with the IF of respiration data as the input feature

\begin{tabular}{cccccc}
\hline Testing & 1 & 2 & 3 & 4 & 5 \\
\hline Accuracy & 1.00 & 0.25 & 0.5 & 0.75 & 0.75 \\
\hline Testing & 6 & 7 & 8 & 9 & 10 \\
\hline Accuracy & 1.00 & 0.75 & 1.00 & 0.75 & 1.00 \\
\hline Average accuracy & & 0.78 & & \\
\hline
\end{tabular}

Emotions affect respiratory responses, and respiratory muscular contraction and relaxation drive the thoracic and abdominal wall movements. Compared with the users with LIA, those with HIA exhibited a higher abdominal muscle contraction amplitude and lower abdominal wall movements whilestaring at positive or negative pictures of 3- to 12-s [42]. The respiratory responses positively related to the scores of the CIAS [19], and the respiratory rate was used to predict the craving of individuals with IGD to play online games [28]. Those with HIA are on the Internet excessively, which can cause aches and soreness in the back [2, 3] or hands; they may change their posture to relieve this pain. Therefore, we determined that the instantaneous responses of respiratory muscle clusters, respiratory wall movements, and body movements constitute potential biomedical indexes through which to observe the psychological reaction of the IA group, with the various emotional stimuli eliciting different respiratory responses. These responses can assist in detecting IA symptoms. We also demonstrated that $\mathrm{IF}_{2}$ during the RE video stimuli was an acceptable predictor of IA risk, which was calculated using the ROC. However, this finding was inconsistent with the feature extraction of the XCSR model and can be attributed to several possible explanations. The ROC employs conventional mathematical calculation, but the XCSR method observes the relationship between features using multiple dimension attributes. In addition, the sampling size and parameter setup of the XCSR may influence feature extraction. Therefore, future works should employ more data samples and test different parameters of the XCSR method.

Table 6: Mean \pm standard deviation of IF of respiration

\begin{tabular}{|c|c|c|c|c|}
\hline \multicolumn{2}{|c|}{ Feature } & $\begin{array}{l}\text { LIA with } \\
\text { non-IGD }\end{array}$ & $\begin{array}{l}\text { HIGD with } \\
\text { IGD }\end{array}$ & $\begin{array}{c}\text { not \# } \\
\text { rate }\end{array}$ \\
\hline \multirow[t]{8}{*}{$\overline{\mathrm{LOL}}$} & $\mathrm{IF}_{1}$ & $12.21 \pm 0.15$ & $12.19 \pm 0.19$ & 0.61 \\
\hline & $\mathrm{IF}_{2}$ & $7.42 \pm 0.27$ & $7.32 \pm 0.38$ & 0.67 \\
\hline & $\mathrm{IF}_{3}$ & $3.64 \pm 0.21$ & $3.53 \pm 0.33$ & 0.64 \\
\hline & $\mathrm{IF}_{4}$ & $1.18 \pm 0.20$ & $1.16 \pm 0.20$ & 0.59 \\
\hline & $\mathrm{IF}_{5}$ & $0.35 \pm 0.08$ & $0.34 \pm 0.07$ & 0.64 \\
\hline & $\mathrm{IF}_{6}$ & $0.18 \pm 0.03$ & $0.18 \pm 0.04$ & 0.57 \\
\hline & $\mathrm{IF}_{7}$ & $0.08 \pm 0.02$ & $0.07 \pm 0.02$ & 0.59 \\
\hline & $\mathrm{IF}_{8}$ & $0.04 \pm 0.01$ & $0.04 \pm 0.01$ & 0.58 \\
\hline \multirow[t]{8}{*}{$\mathrm{MS}$} & $\mathrm{IF}_{1}$ & $12.21 \pm 0.20$ & $12.24 \pm 0.15$ & 0.72 \\
\hline & $\mathrm{IF}_{2}$ & $7.33 \pm 0.29$ & $7.33 \pm 0.40$ & 0.56 \\
\hline & $\mathrm{IF}_{3}$ & $3.55 \pm 0.27$ & $3.57 \pm 0.38$ & 0.58 \\
\hline & $\mathrm{IF}_{4}$ & $1.22 \pm 0.28$ & $1.31 \pm 0.30$ & 0.58 \\
\hline & $\mathrm{IF}_{5}$ & $0.35 \pm 0.11$ & $0.36 \pm 0.13$ & 0.49 \\
\hline & $\mathrm{IF}_{6}$ & $0.18 \pm 0.04$ & $0.18 \pm 0.05$ & 0.56 \\
\hline & $\mathrm{IF}_{7}$ & $0.08 \pm 0.02$ & $0.08 \pm 0.03$ & 0.54 \\
\hline & $\mathrm{IF}_{8}$ & $0.04 \pm 0.01$ & $0.04 \pm 0.01$ & 0.55 \\
\hline \multirow[t]{8}{*}{$\mathrm{RE}$} & $\mathrm{IF}_{1}$ & $12.31 \pm 0.17$ & $12.29 \pm 0.14$ & 0.63 \\
\hline & $\mathrm{IF}_{2}$ & $7.74 \pm 0.16$ & $7.61 \pm 0.28^{*}$ & 0.53 \\
\hline & $\mathrm{IF}_{3}$ & $3.93 \pm 0.16$ & $3.81 \pm 0.32$ & 0.56 \\
\hline & $\mathrm{IF}_{4}$ & $1.54 \pm 0.28$ & $1.45 \pm 0.35$ & 0.55 \\
\hline & $\mathrm{IF}_{5}$ & $0.38 \pm 0.09$ & $0.41 \pm 0.17$ & 0.37 \\
\hline & $\mathrm{IF}_{6}$ & $0.25 \pm 0.06$ & $0.21 \pm 0.04$ & 0.56 \\
\hline & $\mathrm{IF}_{7}$ & $0.10 \pm 0.02$ & $0.10 \pm 0.03$ & 0.44 \\
\hline & $\mathrm{IF}_{8}$ & $0.05 \pm 0.01$ & $0.05 \pm 0.02$ & 0.66 \\
\hline
\end{tabular}

$* \mathrm{p}<0.05$ in the Mann-Whitney U test

This study has some limitations. First, the small sample size may lead to overfitting and affect the verification of items in a questionnaire or the feature extraction of physiological responses. Second, the parameter setup of the XCSR was based on empirical studies and must be tested with different values. Third, the physiological responses of participants while watching online game videos may differ from those actively playing online games. Fourth, we observed respiratory responses during $2 \mathrm{~min}$ of emotional stimuli. Future studies can investigate second-bysecond psychological responses under varying window sizes. Fifth, the unequal sample of men and women may affect the analysis of the XCSR. Notwithstanding these limitations, the XCSR model provides researchers with a computer-aided detection method for IA. The verification of items in a questionnaire can assist in the 
design of a questionnaire for experience sampling method and the real-time detection of IA. The extraction of instantaneous respiratory features can provide psychological insight into the development of IA behavior.

Table 7: Predictive accuracy for the IF value in individuals watching online game videos, calculated with the area under the curve (AUC) of the ROC

\begin{tabular}{|c|c|c|c|c|c|}
\hline \multirow{2}{*}{\multicolumn{2}{|c|}{ Feature }} & \multirow{3}{*}{$\frac{\text { AUC }}{0.52}$} & \multirow{3}{*}{$\frac{p \text {-value }}{0.82}$} & \multicolumn{2}{|c|}{ 95\% confidence interval } \\
\hline & & & & \multirow{2}{*}{$\frac{\text { lower bound }}{0.33}$} & \multirow{2}{*}{$\frac{\text { upper bound }}{0.71}$} \\
\hline LOL & $\mathrm{IF}_{1}$ & & & & \\
\hline & $\mathrm{IF}_{2}$ & 0.52 & 0.80 & 0.33 & 0.71 \\
\hline & $\mathrm{IF}_{3}$ & 0.58 & 0.41 & 0.39 & 0.76 \\
\hline & $\mathrm{IF}_{4}$ & 0.53 & 0.74 & 0.35 & 0.72 \\
\hline & $\mathrm{IF}_{5}$ & 0.56 & 0.55 & 0.37 & 0.74 \\
\hline & $\mathrm{IF}_{6}$ & 0.49 & 0.96 & 0.31 & 0.68 \\
\hline & $\mathrm{IF}_{7}$ & 0.63 & 0.17 & 0.45 & 0.81 \\
\hline & $\mathrm{IF}_{8}$ & 0.51 & 0.96 & 0.32 & 0.69 \\
\hline \multirow[t]{8}{*}{$\mathrm{MS}$} & $\mathrm{IF}_{1}$ & 0.56 & 0.52 & 0.33 & 0.71 \\
\hline & $\mathrm{IF}_{2}$ & 0.51 & 0.91 & 0.38 & 0.74 \\
\hline & $\mathrm{IF}_{3}$ & 0.53 & 0.78 & 0.32 & 0.70 \\
\hline & $\mathrm{IF}_{4}$ & 0.59 & 0.35 & 0.33 & 0.72 \\
\hline & $\mathrm{IF}_{5}$ & 0.52 & 0.84 & 0.41 & 0.77 \\
\hline & $\mathrm{IF}_{6}$ & 0.53 & 0.74 & 0.33 & 0.70 \\
\hline & $\mathrm{IF}_{7}$ & 0.55 & 0.61 & 0.34 & 0.72 \\
\hline & $\mathrm{IF}_{8}$ & 0.56 & 0.55 & 0.36 & 0.74 \\
\hline \multirow[t]{8}{*}{$\mathrm{RE}$} & $\mathrm{IF}_{1}$ & 0.54 & 0.63 & 0.36 & 0.73 \\
\hline & $\mathrm{IF}_{2}$ & 0.70 & 0.03 & 0.51 & 0.89 \\
\hline & $\mathrm{IF}_{3}$ & 0.67 & 0.07 & 0.48 & 0.86 \\
\hline & $\mathrm{IF}_{4}$ & 0.64 & 0.14 & 0.45 & 0.82 \\
\hline & $\mathrm{IF}_{5}$ & 0.52 & 0.87 & 0.32 & 0.71 \\
\hline & $\mathrm{IF}_{6}$ & 0.67 & 0.07 & 0.50 & 0.84 \\
\hline & $\mathrm{IF}_{7}$ & 0.58 & 0.41 & 0.39 & 0.76 \\
\hline & $\mathrm{IF}_{8}$ & 0.52 & 0.82 & 0.33 & 0.71 \\
\hline
\end{tabular}

\section{Conclusion}

This study aimed to detect IA using the XCSR model. This model was also applied to verify the items of the CIAS and extract instantaneous respiratory features. The XCSR achieved over 95\% classification accuracy for both the CIAS data and IF of respiration during online game video stimuli. The average accuracy of the 10 -fold cross-validation was also higher than $75 \%$ for both input data. Furthermore, using the XCSR model, the CIAS was modified from a 26-item to 19-item questionnaire and the IFs of respiratory muscle contractions on the abdomen, respiratory wall movements on the abdomen, and body movements were extracted as the IA-related features. The current findings indicated the suitability of the XCSR method as a computer-aided system for real-time IA detection. The extracted psychological symptoms and instantaneous respiratory responses are key features in determining the development of IA. Further studies should collect more samples to validate these findings; design experiments incorporating active gameplay stimuli; and measure the instantaneous psychophysiological responses per second under varying window sizes.

\section{Conflict of Interest}

The authors declare no conflict of interest.

\section{Acknowledgment}

This work was fully supported by the Taiwan Ministry of Science and Technology (Grant Numbers: MOST 105-2221-E-009-159, MOST 105-2634-E-009-003, and MOST 107-2221-E-009-153). As well, all participants in this study were thanked for their time and contributions.

\section{References}

[1] H.-M. Ji, L.-Y. Chen, T.-C. Hsiao, "Real-Time detection of Internet addiction using reinforcement learning system,” in 2019 Genetic and Evolutionary Computation Conference (GECCO), 1-9, 2019, doi:10.1145/3319619.3326882.

[2] K.S. Young, "Internet addiction: The emergence of a new clinical disorder," CyberPsychology and Behavior, 1(3), 237-244, 1998, doi: 10.1089/cpb.1998.1.237.

[3] S.-H. Chen, L.-J. Weng, Y.-J. Su, H.-M. Wu, P.-F. Yang, "Development of Chinese Internet addiction scale and its psychometric study," Chinese Journal of Psychology, 45(3), 279-294, 2003, doi: 10.1037/t44491-000.

[4] C. Chou, L. Condron, J.C. Belland, "A review of the research on Internet addiction,” Educational Psychology Review, 17(4), 363-388, 2005, doi: 10.1007/s10648-005-8138-1.

[5] J.S. Lemmens, P.M. Valkenburg, J. Peter, "Development and validation of a game addiction scale for adolescents,” Media Psychology, 12, 77-95, 2009, doi: 10.1080/15213260802669458.

[6] M. Beranuy, U. Oberst, X. Carbonell, A. Chamarro, "Problematic internet and mobile phone use and clinical symptoms in college students: The role of emotional intelligence,” Computers in Human Behavior, 25, 1182-1187, 2009, doi:10.1016/j.chb.2009.03.001.

[7] H.-H. Shuai, C.-Y. Shen, D.-N. Yang, Y.-F. C. Lan, W.-C. Lee, P. S. Yu, M.-S. Chen, “ A comprehensive study on social network mental disorders detection via online social media mining,” IEEE Transactions on Knowledge and Data Engineering, 30(7), 1212-1225, 2018, doi: 10.1109/TKDE.2017.2786695.

[8] American Psychiatric Association, Diagnostic and statistical manual of mental disorders, fifth edition (DSM-5), American Psychiatric Association Press Inc., Arlington, VA, 2013.

[9] Y.-S. Jo, S.-Y. Bhang, J.-S. Choi, H.-K. Lee, S.-Y. Lee, Y.-S. Kweon, "Clinical characteristics of diagnosis for Internet gaming disorder: comparison of DSM-5 IGD and ICD-11 GD diagnosis,” Journal of Clinical Medicine, 8, 945, 2019, doi:10.3390/jcm8070945.

[10] M. Romano, A. Roaro, F. Re, L.A. Osborne, R. Truzoli, P. Reed, "Problematic internet users' skin conductance and anxiety increase after exposure to the internet," Addictive Behaviors. 75, 70-74, 2017, doi: 10.1016/j.addbeh.2017.07.003.

[11] J. Morahan-Martin, P. Schumacher, "Incidence and correlates of pathological Internet use among college students,” Computers in Human Behavior, 16(1), 13-29, 2000, doi: 10.1016/S0747-5632(99)00049-7.

[12] Y. Sun, Y.-Y. Li, Y.-P. Bao, S.-Q. Meng, Y.-K. Sun, G. Schumann, T. Kosten, J. Strang, L. Lu, J. Shi, "Brief report: Increased addictive Internet and substance use behavior during the COVID-19 pandemic in China," American Journal on Addictions, 29, 268-270, 2020, doi: 10.1111/ajad.13066.

[13] M.C. Zara, L.H. A. Monteiro, "The negative impact of technological advancements on mental health: An epidemiological approach,” Applied Mathematics and Computation, 396, 125905, 2021, doi: 10.1016/j.amc.2020.125905.

[14] N.M. Petry, F. Rehbein, D.A. Gentile, J.S. Lemmens, Hans-Jürgen Rumpf, T. Mößle, G. Bischof, R. Tao, D.S.S. Fung, G. Borges, et al., “An international consensus for assessing Internet gaming disorder using the new DSM-5 approach,” Addiction, 109, 1399-1406, 2014, doi: 10.1111/add.12457.

[15] K.S. Young, Caught in the net: How to recognize the signs of internet addiction--and a winning strategy for recovery: John Wiley \& Sons, 1998.

[16] M. Pawlikowski, C. Altstötter-Gleich, M. Brand, "Validation and psychometric properties of a short version of Young's Internet addiction test," Computers in Human Behavior, 29(3), 1212-1223, 2013, doi: 10.1016/j.chb.2012.10.014.

[17] H. M. Pontes, M.D. Griffiths, “Measuring DSM-5 internet gaming disorder: Development and validation of a short psychometric scale," Computers in Human Behavior, 45, 137-143, 2015, doi: 10.1016/j.chb.2014.12.006.

[18] K.-K. Mak, C.-M. Lai, C.-H. Ko, C. Chou, D.-I. Kim, H. Watanabe, and 
R.C.M. Ho, "Psychometric properties of the revised Chen Internet addiction scale (CIAS-R) in Chinese adolescents," Journal of Abnormal Child Psychology, 42, 1237-1245, 2014, doi: 10.1007/s10802-014-9851-3.

[19] D.-W. Lu, J.-W. Wang, A.C.W Huang, "Differentiation of Internet addiction risk level based on autonomic nervous responses: The Internet-addiction hypothesis of autonomic activity," Cyberpsychology, Behavior, and Social Networking, 13, 371-378, 2010, doi: 10.1089/cyber.2009.0254.

[20] D.-L. Hsieh, T.-C. Hsiao, "Respiratory sinus arrhythmia reactivity of internet addiction addicts in negative and positive emotional states using film clips stimulation,” BioMedical Engineering OnLine, 15, 69, 2016, doi: 1186/s12938-016-0201-2.

[21] P. Reed, M. Romano, F. Re, A. Roaro, L.A. Osborne, C. Viganò, R. Truzoli, "Differential physiological changes following internet exposure in higher and lower problematic internet users,” PLoS ONE, 12(5), e e0178480, 2017, doi: 10.1371/journal.pone.0178480.

[22] J.-S. Chang, E.-Y. Kim, D. Jung, S.-H. Jeong, Y. Kim, M.-S. Roh, Y.-M. Ahn, B.-J. Hahm, "Altered cardiorespiratory coupling in young male adults with excessive online gaming,” Biological Psychology, 110, 159-166, 2015, doi: 10.1016/j.biopsycho.2015.07.016.

[23] S.-J. Hong, D. Lee, J. Park, K. Namkoong, J. Lee, D.P. Jang, J.E. Lee, Y.-C. Jung, and I.Y. Kim, "Altered heart rate variability during gameplay in Internet gaming disorder: The impact of situations during the game," Frontiers in Psychoiatry, 9, 429, 2018, doi: 10.3389/fpsyt.2018.00429.

[24] K. Ioannidis, S.R. Chamberlain, M.S. Treder, F. Kiraly, E.W. Leppink, S.A. Redden, D.J. Stein, C. Lochner, J.E. Grant, "Problematic internet use (PIU): Associations with the impulsive-compulsive spectrum. An application of machine learning in psychiatry,” Journal of Psychiatric Research, 83, 94102, 2016, doi: 10.1016/j.jpsychires.2016.08.010.

[25] M.J. Rho, J.-E. Jeong, J.-W. Chun, H. Cho, D.J. Jung, I.Y. Choi, D.-J. Kim, "Predictors and patterns of problematic Internet game use using a decision tree model,” Journal of Behavioral Addictions, 5(3), 500-509, 2016, doi: 10.1556/2006.5.2016.051.

[26] S. Aggarwal, S. Saluja, V. Gambhir, S. Gupta, S.P.S. Satia, "Predicting likelihood of psychological disorders in PlayerUnknown's Battlegrounds (PUBG) players from Asian countries using supervised machine learning,” Addictive Behaviors, 101, 106132, 2020, doi: 10.1016/j.addbeh.2019.106132.

[27] D. Seth, S. Eswaran, T. Mukherjee, M. Sachdeva, "A deep learning framework for ensuring responsible play in skill-based cash gaming," in 2020 IEEE International Conference on Machine Learning and Applications (ICMLA), 454-459, 2020, doi: 10.1109/ICMLA51294.2020.00077.

[28] H. Kim, J. Ha, W.-D. Chang, W. Park, L. Kim, C.-H. Im, "Detection of craving for gaming in adolescents with Internet gaming disorder using multimodal biosignals,” Sensors, 18, 102, 2018, doi:10.3390/s18010102.

[29] S.W. Wilson, "Classifier fitness based on accuracy," Evolutionary Computation, 3, 149-175, 1995, doi: 10.1162/evco.1995.3.2.149.

[30] S.W. Wilson, Get real! XCS with continuous-valued inputs. Lanzi et al. (eds) Learning Classifier Systems: From Foundations to Applications. SpringerVerlag, Berlin, 1813 of Lecture Notes in Artificial Intelligence, 209-219,

\section{0.}

[31] H.-M. Ji, T.-C. Hsiao, "A novel cue-induced abdominal reaction analysis for Internet gaming disorder,” Journal of Medical Systems, 43, 94, 2019, doi: 10.1007/s10916-019-1221-9.

[32] M. Griffiths, “Nicotine, tobacco and addiction,” Nature, 384, 18, 1996, doi: 10.1038/384018a0.

[33] V.A. Santos, R. Freire, M. Zugliani, P. Cirillo, H.H. Santos, A.E. Nardi, A.L. King, "Treatment of Internet addiction with anxiety Disorders: Treatment protocol and preliminary before-after results involving pharmacotherapy and modified cognitive behavioral therapy,” JMIR Research Protocols, 5, e46, 2016, doi: 10.2196/resprot.5278.

[34] W. Li, E.L. Garland, J.E. OBBrien, C. Tronnier, P. McGovern, B. Anthony, M.O. Howard, "Mindfulness-oriented recovery enhancement for video game addiction in emerging adults: preliminary findings from case reports," International Journal of Mental Health Addiciton, 16, 928-945, 2018, doi: 10.1007/s11469-017-9765-8.

[35] A.R. Melero Ventola, J.R. Yela, A. Crego, M. Cortés-Rodríguez, "Effectiveness of a mindfulness-based cognitive therapy group intervention in reducing gambling-related craving,” 20(1), 107-134, 2020, doi: 10.24193/jebp.2020.1.7.

[36] N.E. Huang, Z. Shen, S.R. Long, M.C. Wu, H.H. Shih, Q. Zheng, N.-C. Yen, et al., "The empirical mode decomposition and the Hilbert spectrum for nonlinear and non-stationary time series analysis," Proceedings of the Royal Society A, 454(1971), 903-995, 1998, doi: 10.1098/rspa.1998.0193.

[37] Z. Wu, N.E. Huang, "Ensemble empirical mode decomposition: a noiseassisted data analysis method," Advances in Adaptive Data Analysis, 1, 141, 2009, doi: 10.1142/S1793536909000047.

[38] Y.-C. Chen, T.-C. Hsiao, "Towards estimation of respiratory muscle effort with respiratory inductance plethysmography signals and complementary ensemble empirical mode decomposition," Medical \& Biological Engineering \& Computing, 56(7), 1293-1303, 2018. doi: 10.1007/s11517017-1766-z.

[39] N.E. Huang, Z. Wu, S.-R. Long, K.C. Arnold, X. Chen, K. Blank, "On instantaneous frequency,” Advances in Adaptive Data Analysis, 1, 177-229, 2009, doi: 10.1142/S1793536909000096.

[40] M.V. Butz, S.W. Wilson, An algorithmic description of XCS. In Lanzi, P., Stolzmann, W., and Wilson, S., Ed., Advances in Learning Classifier Systems. IWLCS 2000. Lecture Notes in Computer Science, vol 1996. Springer, Berlin, Heidelberg, 253-272, 2001, doi: 10.1007/3-540-44640$0 \_15$.

[41] Y.-H. Lin, Y.-C. Pan, S.-H. Lin, S.-H. Chen, "Development of short-form and screening cutoff point of the smartphone addiction inventory (SPAI-SF)," International Journal of Methods in Psychiatric Research, 26(2), e1525, 2017, doi: $10.1002 / m p r .1525$.

[42] D.-L. Hsieh, H.-M. Ji, T.-C. Hsiao, B.-S. Yip, "Respiratory feature extraction in emotion of Internet addiction abusers using complementary ensemble empirical mode decomposition,” Journal of Medical Imaging and Health Informatics, 5, 1-9, 2015, doi: 10.1166/jmihi.2015.1405. 